\title{
Diagnostic value of bronchoalveolar lavage fluid cryptococcal antigen-lateral flow immunochromatographic assay for pulmonary cryptococcosis in non-HIV patients
}

\section{Hui-Qing Zeng}

Zhongshan Hospital Xiamen University

\section{Xue-Ying Cai}

Zhongshan Hospital Xiamen

Xiao-Bin Zhang ( $\square$ zhangxiaobincn@xmu.edu.cn )

Zhongshan Hospital Xiamen University https://orcid.org/0000-0002-5155-8560

\section{Dong-Yong Yang}

Second Affiliated Hospital of Fujian Medical University

\section{Li Lin}

Zhangzhou Municipal Hospital of Fujian Province and Zhangzhou Affiliated Hospital of Fujian Medical University

\section{Mei-Jun Chen}

Zhongshan Hospital Xiamen University

\section{Wei-Feng Guo}

Fujian Medical University Affiliated First Quanzhou Hospital

\section{Xiongbiao Luo}

\section{Xiamen Univeristy}

\section{Research article}

Keywords: colloidal gold immunochromatographic assay, cryptococcal capsular polysaccharide antigen, bronchoalveolar lavage fluid, pulmonary cryptococosis

Posted Date: September 11th, 2020

DOl: https://doi.org/10.21203/rs.3.rs-50575/v2

License: (c) (i) This work is licensed under a Creative Commons Attribution 4.0 International License. Read Full License 
Version of Record: A version of this preprint was published at Diagnostic Microbiology and Infectious Disease on March 1st, 2021. See the published version at https://doi.org/10.1016/j.diagmicrobio.2020.115276. 


\section{Abstract}

Background: Early diagnosis of pulmonary cryptococcosis (PC) without human immunodeficiency virus (HIV) infection may be challenging. The aim of this study was to investigate the diagnostic value of cryptococcal antigen-lateral flow immunochromatographic assay (CrAg-LFA) in bronchoalveolar lavage fluid (BALF) of patients with PC.

Methods: A total pf 308 patients who were admitted to the departments of pulmonary and critical care medicine and thoracic surgery between March 2015 and October 2018, were divided into the PC group $(n=72)$ and the non-PC group ( $n=236)$. PC was confirmed by 1 ) lung biopsy; 2 ) a positive culture of Cryptococcus neoformans (C. neoformans) from blood culture or biopsy specimen; 3 ) clinical data, pathogen detection, radiological imaging and the detection of the cryptococcal antigen in blood and BALF samples.

Results: The sensitivity, specificity, positive and negative predicted values of CrAg-LFA in the serum were $75.0 \%, 99.6 \%, 98.2 \%$, and $92.9 \%$, respectively, while those in the BALF were $93.1 \%, 100.0 \%, 100.0 \%$, and $97.9 \%$, respectively. The sensitivity of the CrAg-LFA in BALF was significantly higher than that in the serum of the patients in the $P C$ group $(P<0.05)$.

Conclusion: CrAg-LFA is a rapid, simple, and safe experimental method. CrAg-LFA has a higher diagnostic value for PC when analyzing BALF samples compared to serum samples. Furthermore, the BALF positive results are equivalent to the microbiological culture-positive results in terms of diagnostic value.

\section{Introduction}

Pulmonary cryptococcosis (PC) is a subacute or chronic lung disease caused by Cryptococcus neoformans infection, which is commonly found in immunocompetent patients [1]. The diagnosis of PC is still challenging because the clinical manifestations and imaging of PC have no prominent characteristics compared with other pulmonary diseases[2]. , the diagnosis of PC is mainly based on pathological examination and culture of biopsy specimens obtained from sterile sites; yet, these methods are invasive and require time.

The detection of cryptococcal capsular polysaccharide antigen in serum has important clinical significance in the diagnosis of PC. The new cryptococcal antigen-lateral flow assay (CrAg-LFA), which has been recently developed, is easy to perform and has an advantage of rapid detection. The simultaneous detection of galactomannan antigen (GM) in the serum and bronchoalveolar lavage fluid (BALF) for the diagnosis of pulmonary aspergillosis can confirm that GM sensitivity of BALF is significantly higher than that of serum GM [3]. Nonetheless, the effectiveness of cryptococcal capsular polysaccharide antigen in BALF, notably using CrAg-LFA, in the diagnosis of PC, has not yet been reported. In the present study, CrAg-LFA was used to detect the cryptococcal antigen in BALF and serum samples. This evidence was combined with the clinical-pathological outcomes, so as to explore its diagnostic value for PC. 


\section{Subjects And Methods}

\section{Subjects}

From March 2015 to October 2018, patients who were admitted to the departments of pulmonary and critical care medicine and thoracic surgery were screened consecutively. These departments were based in the Zhongshan Hospital, Xiamen University, the Zhangzhou affiliated hospital of the Fujian Medical University, the Second affiliated Hospital of the Fujian Medical University, and the Quanzhou First Hospital affiliated to the Fujian Medical University. The patients who exhibited nodular lesions near the lungs, had lung shadows, and were suspected of PC following ineffective general antibacterial therapy were included in our study.

This study was approved by the Ethics committees of Zhongshan Hospital, Xiamen University (approved no. 2017030). All patients signed informed consent forms.

\section{Instruments and reagents}

Cryptococcus antigen detection kit (CrAg-LFA detection) was a product of Immuno Mycologics, USA. The fungal identification card YST and the automated blood culture system Bact/Alert3D were purchased from Biomerieux Ltd., France. The BACTEC FX automated blood culture system was purchased from Becton Dickinson, USA.

\section{Pathological examination}

Percutaneous lung biopsy was guided by CT or B-ultrasound and tissue biopsy was conducted using an electronic bronchoscopy. Conventional tissue fixation that included embedding and sectioning were performed by researchers who were blinded to the origin of the samples. All samples were subject to H\&E staining and PAS, as well as special staining, such as hexamine silver.

\section{Grouping}

The human immunodeficiency virus (HIV) antibody was detected, and the negative result was confirmed in all enrolled patients. All patients were divided into two groups: (1) The PC group included patients who were diagnosed as PC and underwent serum and BALF CrAg-LFA, as well as microbial culture prior to antifungal treatment; (2) The patients of the non-PC group provided blood samples, bronchoscopy results, and BALF samples for the assessment of the diagnostic procedure.

All PC patients were confirmed using (1) lung tissues pathological evidence through percutaneous lung biopsy or transbronchial lung biopsy (non-caseating granulomas combined with devoured cryptococci cells and capsules in the cytoplasm of macrophages); (2) positive culture of $C$. neoformans from blood culture or biopsy specimen; [4] clinical data, pathogen detection, radiological imaging and the detection of the cryptococcal antigen in blood and BALF samples, as well as in their microbial culture of all included patients were collected. 
Lumbar puncture was conducted in PC patients in order to rule out asymptomatic central nervous system involvement.

\section{BALF specimen collection}

BALF samples were collected from all included patients before antifungal treatment, according to the standard operating procedure (SOP) using the bronchoscope. Following the induction of local anesthesia with $2 \%$ lidocaine, nasal bronchoscopy was performed. The bronchoscope was inserted into the target sub-segment bronchus for high resolution computed tomography (HRCT) positioning. Then, $30 \mathrm{ml}$ of sterile saline was injected at $37^{\circ} \mathrm{C}$. The injection was performed two times for bronchoalveolar lavage to the lesion site. Subsequently, 15 to $30 \mathrm{ml}$ of the BALF sample was recycled, placed in a silicone plastic bottle, and sent (at room temperature) for detection. The solution was stored for $72 \mathrm{~h}$ at $4^{\circ} \mathrm{C}$.

\section{Microbial culture}

The serum samples were collected from all included patients for blood culture. A total of $5 \mathrm{ml}$ of fasting serum was collected before antifungal treatment, and the supernatant was stored in a refrigerator at $-20^{\circ} \mathrm{C}$. The alarm-positive culture flask was immediately transferred to the blood agar plate and the Shabao's plate to allow growth and separation, followed by identification using the YST card. The BALF or bronchoscopy brush was directly inoculated in the blood agar and/or in the Shabu's plates in order to allow growth and separation, followed by identification using the YST card.

\section{CrAg-LFA}

Using a blinded study design, 5 to $10 \mathrm{ml}$ of the BALF and whole blood sterile samples were routinely collected, from which serum was isolated according to the instructions provided by the kit manufacturer. One drop of the sample dilution was placed into a small test tube and mixed with $40 \mu$ of the separated sample. Subsequently, the white end of a test strip was immersed into the solution; the sample was removed $10 \mathrm{~min}$ later for detection. A negative result was defined by the appearance of one quality control line. In contrast, a positive result was defined by the simultaneous appearance of a quality control line and a test line. A failed result was defined by the appearance of the test line only, which indicated that it required re-testing.

\section{Statistical analysis}

Statistical analyses were performed using SPSS 21.0 software. The data that followed non-normal distribution were analyzed using the Kruskal-Wallis test. The categorical data or grading data were analyzed by the Chi-square test. A P value lower than $0.05(P<0.05)$ was used as statistically significant differences.

\section{Results}


Among the 308 patients, 72 cases were diagnosed with PC. The diagnosis of PC was established in accordance with the standard criteria, as previously described [4]. More than half of PC patients were asymptomatic (40;55.6\%). Fever was more commonly observed in the PC group compared to the non-PC group (31.9\% vs. $17.3 \%, \mathrm{P}=0.008)$. The underlying diseases among the PC group included diabetes mellitus, old tuberculosis, lung cancer, malignancy, hematological disease, pulmonary vasculitis; however, most of the PC patients $(51,70.8 \%)$ were without underlying diseases.

Laboratory analysis suggested that procalcitonin and GM were higher in the non-PC group than in the PC group; other variables, including white blood cells, neutrophil, lymphocyte subsets, were not different between groups (Table 1).

Table 1. Baseline characteristics between PC and non-PC patients

\begin{tabular}{|c|c|c|c|}
\hline Variable & PC group $(n=72)$ & Non-PC group $(\mathrm{n}=236)$ & $\mathrm{P}$ value \\
\hline Male gender, n (\%) & $39(54.2)$ & $146(61.9)$ & 0.541 \\
\hline Age, years & $48.4(35-64)$ & $52.3(38-70)$ & 0.862 \\
\hline \multicolumn{4}{|l|}{ Symptoms } \\
\hline Cough, n (\%) & $32(44.4)$ & $125(60.0)$ & 0.205 \\
\hline Expectoration, n (\%) & $8(11.1)$ & $75(31.8)$ & 0.001 \\
\hline Fever, $\mathrm{n}(\%)^{1}$ & $23(31.9)$ & $41(17.3)$ & 0.008 \\
\hline Dyspnea, n (\%) & $8(11.1)$ & $59(25.0)$ & 0.012 \\
\hline Hemoptysis, n (\%) & $8(11.1)$ & $66(30.0)$ & 0.003 \\
\hline Asymptomatic, n (\%) & $40(55.6)$ & $64(27.1)$ & 0.000 \\
\hline \multicolumn{4}{|l|}{ Underlying diseases } \\
\hline Diabetes mellitus, n (\%) & $6(8.3)$ & $34(14.4)$ & 0.180 \\
\hline Old tuberculosis, n (\%) & $5(6.9)$ & $15(6.3)$ & 0.859 \\
\hline Lung cancer, n (\%) & $5(6.9)$ & $40(16.9)$ & 0.035 \\
\hline Malignancy except lung cancer, n (\%) & $3(4.2)$ & $6(2.5)$ & 0.474 \\
\hline Hematological disease, $\mathrm{n}(\%)$ & $1(1.4)$ & $15(6.4)$ & 0.131 \\
\hline Connective tissue disease, $\mathrm{n}(\%)$ & $0(0.0)$ & $9(3.8)$ & 0.123 \\
\hline Pulmonary vasculitis, n (\%) & $1(1.4)$ & $7(3.0)$ & 0.686 \\
\hline Corticosteroids usage, n (\%) & $6(8.3)$ & $16(6.8)$ & 0.654 \\
\hline Immunosuppressive agent, n (\%) & $4(5.6)$ & $13(5.5)$ & 0.988 \\
\hline \multicolumn{4}{|l|}{ Laboratory findings } \\
\hline White blood cells, $\times 10^{\wedge} 9 / \mathrm{L}$ & $8.85(4.21-12.3)$ & $6.87(3.25-10.58)$ & 0.068 \\
\hline Neutrophil, \% & $78.6(54.3-87.6)$ & $82.5(65.9-91.3)$ & 0.742 \\
\hline Procalcitonin, $\mu \mathrm{g} / \mathrm{L}$ & $0.13(0.04-0.32)$ & $0.21(0.12-0.45)$ & 0.038 \\
\hline CD4+ lymphocyte, counts $/ \mu l$ & $845.2(515.6-1245.4)$ & $798.6(496.8-1145.3)$ & 0.698 \\
\hline CD8+ lymphocyte, counts $/ \mu l$ & $648.5(415.9-1047.9)$ & $705.6(523.5-1125.9)$ & 0.418 \\
\hline Positive $1,3-\beta$-D glucan & $6.1(3.9-8.4)$ & $7.3(4.9-11.6)$ & 0.872 \\
\hline Serum galactomannan & $0.31(0.14-0.45)$ & $0.65(0.41-1.35)$ & 0.021 \\
\hline \multicolumn{4}{|l|}{ CT images } \\
\hline Single nodule, n (\%) & $21(29.2)$ & $62(26.3)$ & 0.628 \\
\hline Multiple nodules, n (\%) & $32(44.4)$ & $84(35.6)$ & 0.175 \\
\hline Diffuse pulmonary parenchymal changes, n (\%) & $7(9.7)$ & $89(37.7)$ & 0.000 \\
\hline Two lungs involved, $\mathrm{n}(\%)$ & $37(51.4)$ & $158(66.9)$ & 0.016 \\
\hline Cavities or vacuoles & $9(12.5)$ & $31(13.1)$ & 0.888 \\
\hline Mediastinal lymph node, n (\%) & $7(9.7)$ & $26(11.0)$ & 0.756 \\
\hline Pleural effusion, $\mathrm{n}(\%)$ & $6(8.3)$ & $38(16.1)$ & 0.099 \\
\hline Positive serum microbial culture ${ }^{2}$ & $1(1.4)$ & $0(0.0)$ & 0.234 \\
\hline Positive BALF microbial culture $^{2}$ & $9(12.5)$ & $0(0.0)$ & 0.000 \\
\hline
\end{tabular}

Abbreviation: PC, pulmonary cryptococcosis, CT, computer tomography, BALF, bronchoalveolar lavage fluid. 1: Temperature was high than $37.3^{\circ} \mathrm{C} .^{2}$ : Cryptococcus. Neoformans. 
Among the 72 PC patients, the lesions were localized in the peripheral lung fields. Both lungs were involved in 66 PC cases. The lesions were localized in the basal lobes in 37 cases. Besides, 21 cases of single nodules, and 32 cases of multiple nodules, including 21 cases of the right lung and 14 cases of the left lung, were observed. A total of 8 cases showed multiple plaques; 15 lump cases had multiple nodules, and 7 cases of diffuse pulmonary parenchymal changes were diagnosed. A total of 9 cases had cavities or vacuoles in their lesions, 7 cases presented with enlargement of mediastinal lymph node, and 6 cases exhibited pleural effusion (Table 1).

\section{Diagnostic efficiency of cryptococcal antigen and culture in serum and BALF for PC}

The CrAg-LFA data and the blood culture results from serum and BALF of the PC and non-PC groups are shown in Tables 2 and 3, respectively.

Table 2. Cryptococcal antigen and culture results in the serum

\begin{tabular}{llllll}
\hline Group & \multirow{2}{*}{ Number of cases } & \multicolumn{2}{l}{ Cryptococcal antigen } & \multicolumn{2}{l}{ Culture (Cryptococcus) } \\
\cline { 3 - 6 } & & Positive & Negative & Positive & Negative \\
\hline PC & 72 & $54(75.0)$ & $18(25.0)$ & $1(1.4)$ & $71(98.6)$ \\
Non-PC & 236 & $1(0.4)$ & $235(99.6)$ & $0(0.0)$ & $97(100.0)$ \\
Sum & 308 & 55 & 253 & 1 & 168 \\
$x^{2}$ & & 209.177 & & & \\
P-value & $<0.001$ & & P $=0.426^{*}$ & \\
\hline
\end{tabular}

*: Fisher exact probability method was used since the expected count of two cells is $<5$

Table 3. Cryptococcal antigen and culture results in BALF

\begin{tabular}{llllll}
\hline Group & \multirow{2}{*}{ Number of cases } & \multicolumn{2}{c}{ Cryptococcal antigen } & \multicolumn{2}{l}{ Culture (Cryptococcus) } \\
\cline { 3 - 6 } & & Positive & Negative & Positive & Negative \\
\hline PC & 72 & $67(93.1)$ & $5(6.9)$ & $9(12.5)$ & $63(87.5)$ \\
Non-PC & 236 & $0(0.0)$ & $236(100.0)$ & $0(0.0)$ & $121(100.0)$ \\
Sum & 308 & 67 & 241 & 9 & 184 \\
$X^{2}$ & & 280.665 & & & \\
P-value & $<0.000$ & & $<0.000^{*}$ & \\
\hline
\end{tabular}

*: Fisher exact probability method was used since the expected count of one cell is $<5$

Only one PC patient (1.4\%) had a positive serum cryptococcal culture. With regard to the serum CrAg-LFA data, the sensitivity, specificity, positive predictive value, and negative predictive value were $75.0 \%, 99.6 \%$, $98.2 \%$, and $92.9 \%$, respectively. Nine PC patients (12.5\%) had a positive cryptococcal culture in BALF.

About the BALF CrAg-LFA data, the sensitivity, specificity, positive predictive value, and negative predictive value were $93.1 \%, 100.0 \%, 100.0 \%$, and $97.9 \%$, respectively. The PC group exhibited a significantly higher sensitivity to BALF CrAg-LFA than that noted for the serum CrAg-LFA $(P<0.05)$ (Table 4 and Table 5).

Table 4. Comparison of cryptococcal antigen detection from serum and BALF

\begin{tabular}{llllll}
\hline Group & \multirow{2}{*}{ Number of cases } & \multicolumn{2}{c}{ Serum } & \multicolumn{2}{c}{ BALF } \\
\cline { 3 - 6 } & & Positive & Negative & Positive & Negative \\
\hline PC & 72 & $54(75.0)$ & $18(25.0)$ & $67(93.1)$ & $5(6.9)$ \\
Non-PC & 236 & $1(0.4)$ & $235(99.6)$ & $0(0.0)$ & $236(100.0)$ \\
Sum & 308 & 55 & 253 & 67 & 241 \\
$X^{2}$ & & 209.177 & & 280.665 & \\
P-value & $<0.001$ & & $<0.001$ & \\
\hline
\end{tabular}


Abbreviation: BALF, bronchoalvolar lavage fluid, PC: pulmonary cryptococcosis

Table 5. Detection of cryptococcal antigen in serum and BALF samples in the PC group (\%)

\begin{tabular}{llll}
\hline Group & Serum group & BALF group & P-value \\
& & & \\
\hline Sensitivity & 75.0 & 93.1 & $<0.05$ \\
Specificity & 99.6 & 100.0 & $>0.05$ \\
Positive predictive value & 98.2 & 100.0 & $>0.05$ \\
Negative predictive value & 92.9 & 97.9 & $<0.05$ \\
\hline
\end{tabular}

\section{Discussion}

Due to non-specific clinical features, the diagnosis of PC mainly depends on pathological examination, i.e, lung tissue pathological biopsy, H\&E staining, PAS, and hexamine silver staining [5]. The type of lesion is related to the immune status of the patients. Non-caseating granulomas are often formed in patients with normal immune function and consist of devoured cryptococci cells and capsules that are visible in the cytoplasm of macrophages. Granulomas do not commonly appear in patients with impaired immune function, although the alveolar cavity is filled with cryptococci spores. Specialized bacterial staining can be used to indicate PAS (+), AB (-), hexamine silver (+), and acid resistance (-). C. neoformans is mainly detected using blood culture or punctured materials. The aforementioned examinations have a long reporting time and a low positive rate. In addition, these methods are invasive, thus unsuitable for critically ill patients and patients with blood coagulation dysfunction. Moreover, these examinations have limited value for early diagnosis of the disease.

Over recent years, the determination of cryptococcal polysaccharide capsular antigen in serum samples has provided etiological evidence for the diagnosis of PC. Currently, the methods for detecting the cryptococcal capsular antigen in serum include latex agglutination (LA), enzyme-linked immunosorbent assay (EIA), and lateral chromatography (CrAg-LFA) [6]. A previous study has shown that CrAg-LFA exhibited higher accuracy than LA and EIA, resulting in a significant clinical value. This conclusion was derived due to its optimal stability at room temperature, the rapid detection procedure (10 $\mathrm{min}$ ), the ease of operation, and its limited high demand for laboratory equipment [7].

A large number of clinical studies have proven that the sensitivity, specificity, and clinical application value of GM detection in BALF samples (target lung lesion area) are significantly higher than those noted in serum for the diagnosis of invasive pulmonary aspergillosis $[8,9]$. In this study, we extracted BALF samples at the target lesion of PC patients. Thus we compared the CrAg-LFA sensitivity and specificity between BALF and serum samples. The results demonstrated that the sensitivity and specificity of CrAgLFA on BALF samples were $93.1 \%$ and $100.00 \%$, respectively, while those of serum samples were $75.0 \%$ and $99.6 \%$, respectively, which suggested that the diagnostic value of CrAg-LFA in detecting PC infection is higher when using BALF samples; this was consistent with a previous report [10]. 
In the present study, the colloidal gold method was used to test the cryptococcal capsular polysaccharide antigen. The colloidal gold test strip comprises a rapid and straightforward detection reagent that can detect all four (type A-D) serotypes of $C$. neoformans. During the test, the sample is subjected to capillary action and is chromatographed, by binding to a monoclonal antibody against the Cryptococcus antigen, which is considered a gold standard detection method. In the presence of cryptococcal antigen, binding to the gold standard anti-Cryptococcus antibody occurs. This gold standard antibody-antigen complex continues to be chromatographed on the membrane by capillary action and reacts with the test strip containing immobilized anti-Cryptococcus monoclonal antibody. The cryptococcal capsular polysaccharide antigen in the test sample can react with the colloidal gold-labeled antibody, and the antigen-antibody complex migrates on the surface of the test paper under capillary action and ultimately binds to the monoclonal antibody immobilized on the nitrocellulose membrane. This binding produces a red line that is considered the test line [11]. The test strip itself carries a quality control line. A positive result denotes the simultaneous appearance of the quality control line and the test line, and a negative result denotes the appearance of the quality control line alone. No negative control is required, and this method is considered more reliable.

In the present study, 13 of the 18 pathologically confirmed PC patients who had negative serum CrAg-LFA had positive BALF CrAg-LFA. In certain patients, no clinical symptoms appeared, although small lesions were found at the edge of the lung and even near to the pleura of the lung as determined by CT. In one case with bronchial Cryptococcus, the lesion was found in the lateral bronchus of the right middle lobe. The serum CrAg-LFA test for this case was negative, while the bronchial flushing fluid CrAg-LFA test was positive. It has been shown that the false-negative results of the capsular polysaccharide antigen detection occur mostly during the early stages of infection [12]. The higher positive rate and sensitivity noted in the BALF samples as opposed to the serum samples may be due to the infiltration of cryptococci colonies to the lung, resulting in a slow release of the capsular polysaccharide antigen to the blood. These events occur in the early stage of infection, notably in patients without systemic symptoms. Besides, the infection is associated with a high local cryptococcal load noted in the lung lesions. Yet, these speculations need further confirmation. In the present study, both the serum and BALF results of 5 confirmed cases were negative and corresponded to patients with small lesions and no symptoms. No case was found to have positive serum CrAg-LFA results and negative BALF CrAg-LFA results.

Bronchoalveolar lavage is known as liquid lung biopsy. It is a simple, safe, and convenient compared to the histopathological biopsy that can be implemented in primary hospitals. It can be used for cryptococcal antigen detection in case of biopsy unavailability. However, the process of collecting BALF and the requirements of the operators need to be further standardized. The procedure is complex and requires high operational skills. In addition, it may not be appropriate for small lesions. In the present study, the location of BALF collection was accurately positioned using lung CT, and only $60 \mathrm{ml}$ of liquid was perfused to avoid the dilution of specimens caused by excessive perfusion. Since less than $1 \mathrm{ml}$ of fluid is required for CrAg-LFA, this method can increase the positive rate by a collection of the centrifuged precipitation of the lavage fluid. 
The CrAg-LFA test exhibits significantly higher sensitivity, specificity, and application value in BALF than in serum samples in a similar manner with the GM. However, GM may generate false-positive results when testing both serum and BALF samples and can be influenced by several factors $[13,14]$. It has been reported that both LA and colloidal gold methods can occasionally lead to false-positive results with regard to the detection of serum cryptococcal capsular polysaccharide antigen. In the present study, one out of 236 cases of non-PC resulted as weakly positive with regard to the serum CrAg-LFA test, which suggested that specific factors cause potential cross-reactivity in case of a low serum cryptococcal antigen titer. Therefore, a semi-quantitative determination is advised in such cases. Finally, this case was pathologically diagnosed as rheumatoid lung disease. Whether such rare diseases cross-react with serum cryptococcal antigen to a certain extent needs to be confirmed by large sample clinical trials. In the present study, no false-positive results were found in BALF samples from the four centers tested by CrAgLFA, and the test exhibited a specificity of $100.00 \%$. Therefore, our data suggest that the diagnostic value of the CrAg-LFA test in BALF samples has equivalent positive performance in detecting cryptococcal antigen as the microbiological culture, and it is only inferior to the clinical value of the histopathological biopsy. Furthermore, lung puncture and other traumatic examinations are required only in cases with negative results.

This study has a few limitations. First, we did not compare the diagnostic efficiency between LFA and conventional methods, such as LA or EIA. Second, the BALF CrAg-LFA value after treatment was not detected; therefore, we failed to evaluate the effectiveness of anti-cryptococcal therapy.

In summary, CrAg-LFA is a rapid, simple, and safe experimental method, which can be used for early diagnosis of PC with small lesions. CrAg-LFA has a higher diagnostic value for PC when analyzing BALF samples compared to serum samples. Furthermore, the BALF positive results are equivalent to the microbiological culture-positive results in terms of diagnostic value.

\section{Abbreviations}

BALF: bronchoalveolar lavage fluid

CrAg-LFA: cryptococcal antigen-lateral flow assay

EIA: enzyme-linked immunosorbent assay

GM: galactomannan

HIV: Human immunodeficiency virus

HRCT: high resolution computed tomography

LA: latex agglutination

PC: Pulmonary cryptococcosis

Page 10/13 
SOP: standard operating procedure

\section{Declarations}

\section{Ethics approval and consent to participate}

This study was approved by the Ethics committees of Zhongshan Hospital, Xiamen University (approved no. 2017030). All patients signed informed consent forms.

\section{Consent to publish}

No applicable.

\section{Availability of data and materials}

All the data supporting our findings are contained within this article.

\section{Competing interests}

All authors declare that they have no conflict of interests.

\section{Funding}

This study was supported by the Guiding Project of Science and Technology Plan of the Science and Technology Bureau, Fujian province, China (2017D0016), by the Youth Research Fund from Fujian Provincial Health Bureau (Grant 2018-2-65), and the Fund from Natural Science Foundation of Fujian Province, China (Grant 2018J01393).

\section{Authors' contributions}

(I) Conception and design: Hui-Qing Zeng, Xue-Ying Cai, Xiao-Bin Zhang

(II) Administrative support: Hui-Qing Zeng, Xiongbiao Luo

(III) Provision of study materials or patients: Hui-Qing Zeng, Xue-Ying Cai

(IV) Collection and assembly of data: Hui-Ying Zeng, Xiao-Bin Zhang, Dong-Yong Yang, Li Lin

(V) Data analysis and interpretation: Hui-Qing Zeng, Xue-Ying Cai, Xiao-Bin Zhang, Dong-Yong Yang, Li Lin, Mei-Jun Chen, Wei-Feng Guo, Xiongbiao Luo

(VI) Manuscript writing: All authors

(VII) Final approval of manuscript: All authors

\section{Acknowledgements}


No applicable.

\section{References}

1. Jarvis JN, Harrison TS: Pulmonary cryptococcosis. Semin Respir Crit Care Med 2008, 29(2):141-150.

2. Ye F, Xie JX, Zeng QS, Chen GQ, Zhong SQ, Zhong NS: Retrospective analysis of 76 immunocompetent patients with primary pulmonary cryptococcosis. Lung 2012, 190(3):339-346.

3. Zhou W, Li H, Zhang Y, Huang M, He Q, Li P, Zhang F, Shi Y, Su X: Diagnostic Value of Galactomannan Antigen Test in Serum and Bronchoalveolar Lavage Fluid Samples from Patients with Nonneutropenic Invasive Pulmonary Aspergillosis. J Clin Microbio/ 2017, 55(7):2153-2161.

4. Perfect JR, Dismukes WE, Dromer F, Goldman DL, Graybill JR, Hamill RJ, Harrison TS, Larsen RA, Lortholary $\mathrm{O}, \mathrm{Nguyen} \mathrm{MH}$ et al: Clinical practice guidelines for the management of cryptococcal disease: 2010 update by the infectious diseases society of america. Clin Infect Dis 2010, 50(3):291322.

5. Zhang Y, Li N, Zhang Y, Li H, Chen X, Wang S, Zhang X, Zhang R, Xu J, Shi J et al: Clinical analysis of 76 patients pathologically diagnosed with pulmonary cryptococcosis. Eur Respir J 2012, 40(5):11911200.

6. Saha DC, Xess I, Biswas A, Bhowmik DM, Padma MV: Detection of Cryptococcus by conventional, serological and molecular methods. J Med Microbiol 2009, 58(Pt 8):1098-1105.

7. Lindsley MD, Mekha N, Baggett HC, Surinthong Y, Autthateinchai R, Sawatwong P, Harris JR, Park BJ, Chiller T, Balajee SA et al: Evaluation of a newly developed lateral flow immunoassay for the diagnosis of cryptococcosis. Clin Infect Dis 2011, 53(4):321-325.

8. Zhang XB, Chen GP, Lin QC, Lin X, Zhang HY, Wang JH: Bronchoalveolar lavage fluid galactomannan detection for diagnosis of invasive pulmonary aspergillosis in chronic obstructive pulmonary disease. Med Mycol 2013, 51(7):688-695.

9. Fortun J, Martin-Davila P, Gomez Garcia de la Pedrosa E, Silva JT, Garcia-Rodriguez J, Benito D, Venanzi E, Castano F, Fernandez-Ruiz M, Lazaro F et al: Galactomannan in bronchoalveolar lavage fluid for diagnosis of invasive aspergillosis in non-hematological patients. $J$ Infect 2016, 72(6):738744.

10. Oshima K, Takazono T, Saijo T, Tashiro M, Kurihara S, Yamamoto K, Imamura Y, Miyazaki T, Tsukamoto M, Yanagihara $\mathrm{K}$ et al: Examination of cryptococcal glucuronoxylomannan antigen in bronchoalveolar lavage fluid for diagnosing pulmonary cryptococcosis in HIV-negative patients. Med Mycol 2018, 56(1):88-94.

11. Boulware DR, Rolfes MA, Rajasingham R, von Hohenberg M, Qin Z, Taseera K, Schutz C, Kwizera R, Butler EK, Meintjes $G$ et al: Multisite validation of cryptococcal antigen lateral flow assay and quantification by laser thermal contrast. Emerg Infect Dis 2014, 20(1):45-53.

12. Perfect JR, Bicanic T: Cryptococcosis diagnosis and treatment: What do we know now. Fungal Genet Biol 2015, 78:49-54. 
13. Park SY, Lee SO, Choi SH, Sung H, Kim MN, Choi CM, Hong SB, Oh YM, Shim TS, Koh Y et al: Aspergillus galactomannan antigen assay in bronchoalveolar lavage fluid for diagnosis of invasive pulmonary aspergillosis. J Infect 2010, 61(6):492-498.

14. Boonsarngsuk V, Niyompattama A, Teosirimongkol C, Sriwanichrak K: False-positive serum and bronchoalveolar lavage Aspergillus galactomannan assays caused by different antibiotics. Scand $\mathrm{J}$ Infect Dis 2010, 42(6-7):461-468.

\section{Supplementary Files}

This is a list of supplementary files associated with this preprint. Click to download.

- eTable1cerebrospinalfluidexaminationrevised.pdf 